\title{
MULTI-MODE MULTI-DIMENSIONAL SYSTEMS WITH POISSONIAN SEQUENCING*
}

\author{
ERIK I. VERRIEST ${ }^{\dagger}$
}

\begin{abstract}
The dynamics of hybrid systems with mode dynamics of different dimensions is described. The first part gives some deterministic examples of such multi-mode multi-dimensional $\left(M^{3} D\right)$ systems. The second part considers such models under sequential switching at random times. More specifically, the backward Kolmogorov equation is derived, and Lie-algebraic methods are used in the case where the modes are linear. For Poissonian switched equi-dimensional modes, the diffusion limit and its implication in vibrational stability are studied. The motion of a pebble on an elevator belt is given as an example.
\end{abstract}

Keywords: Hybrid Systems, Optimal Control, Switched Systems, Model Reduction

1. Introduction. We consider a hybrid system with inputs and outputs. Such a system exhibits different modes of operation, but we assume in our model that the different modes of operation have a behavior that can be captured by a state space model perhaps exhibiting different dimensions. We coined the name multi-modal multi-dimensional $\left(M^{3} D\right)$ system for this class. A new feature of such a model is that, at the switching instants, the mapping of the states from old mode to new mode needs to be specified. This class of systems was introduced in [24] where the optimal timing (switching) control was studied. This $M^{3} D$ problem is of interest in the approximate and reduced order modelling for nonlinear systems, as for instance obtained from piecewise linearization or nonlinear balancing [25]. However, several other instances of such systems occur rather naturally, e.g., the dynamics of a trampoline jumper, trapeze circus artists, or simply the motion of material (a slurry) on a transport band and the Brownian motor (nanoscale motion of myosin) $[4,5]$ among many others. Switched systems belong to a general class of hybrid systems, discussed in [1, 2, 22]. In this paper we consider a stochastic $M^{3} D$ problem, where the system cycles through the modes in sequential fashion, repeating over and over, but with the switching times governed by a Poison process $\left(P M^{3} D\right)$.

The paper is organized as follows: In section 2, we describe the system dynamics, and sketch some instances where such models may occur. In Section 3 we consider stochastic switching and derive a hyperbolic PDE to describe the behavior of the system under randomly timed switching, and describe its limit under fast switching in the equi-dimensional case. Finally, Section 4 extends these results to the $P M^{3} D$

\footnotetext{
*Dedicated to Roger Brockett on the occasion of his 70th birthday.

${ }^{\dagger}$ Erik I. Verriest is with the Faculty of Electrical and Computer Engineering, Georgia Institute of Technology, Atlanta, GA 30332-0250. E-mail: erik.verriest@ece.gatech.edu, presently on leave at SCD, ESAT, KULeuven, Leuven, Belgium
} 
case.

2. Dynamics of $M^{3} D$ Systems. The dynamical system discussed in this paper is a switched mode system where we assume that the control on the system consists of a continuous time input $u(\cdot)$ and the switching times, $\left\{\tau_{i}\right\}$. In the stochastic problem, the latter are random and modeled as Poisson arrival times. For simplicity, we assume in this paper that the different modes (controlled vector fields from the set $\left\{f^{(a)}\left(x^{(a)}, u\right)\right\}$, with $\left.a \in \Xi\right)$, are sequenced in periodic order. The output in mode $a$ is $y=h^{(a)}\left(x^{(a)}\right)$. It will be assumed that in each mode, the system may have different order. Thus, let $\operatorname{dim} x^{(a)}=n^{(a)}{ }^{1}$

It will be advantageous to introduce $\xi(t)$, a discrete state, taking values in the finite set, $\Xi$, to denote the mode operating at time $t$. If $\xi(t)=a \in \Xi$, then the dynamical system at $t$ is given by the system in state space form

$$
\dot{x}^{(a)}(t)=f^{(a)}\left(x^{(a)}, u\right), \quad y=h^{(a)}\left(x^{(a)}, u\right) .
$$

with $x^{(a)}$ the state in the state space of mode $a$.

It is then simpler to parameterize the state by the sequential index rather than the identifier in $\Xi$. Thus if $t$ is in the interval $\left(\tau_{i-1}, \tau_{i}\right)$, set

$$
\begin{aligned}
x^{(\xi(t))} & =x_{i}(t), \\
f^{(\xi(t))}(x(t), u(t)) & =f_{i}\left(x_{i}(t), u(t)\right), \\
h^{(\xi(t))}(x(t), u(t)) & =h_{i}\left(x_{i}(t), u(t)\right)
\end{aligned}
$$

The subscript $i$ is not the $i$-th component of $x$ in this paper!

2.1. State Space. In view of the above model, we take the discrete bundle over $\Xi$, with fiber $\mathbb{R}^{n^{(a)}}$ at $a \in \Xi$ as the state space for this multi-mode delay system. The vector $x^{(a)}(t) \in \mathbb{R}^{n^{(a)}}$ is called the partial state at $t$, and $\xi(t) \in \Xi$, the hybrid state (mode variable). The piecewise constant function $\xi(t)$ is restricted to have only finitely many discontinuities in any finite interval. (This holds true in a Poisson switching model)

Sequential switching implies that only the transitions $1 \rightarrow 2 \rightarrow \cdots \rightarrow N \rightarrow 1 \cdots$ are allowed. Hence we should use $[i]=i \bmod N$ as index, but for the sake of simplicity of notation, we'll simply keep $i$. Between switchings, the dynamics in mode $i$ are given by the smooth system

$$
\begin{aligned}
\dot{x}_{i} & =f_{i}\left(x_{i}, u\right) \\
y & =h_{i}\left(x_{i}, u\right)
\end{aligned}
$$

At the switching instants, say from mode $i$ to mode $i+1$, we consider pseudo continuity (defined below).

\footnotetext{
${ }^{1}$ More generally, we could also let the input and output spaces be different in each mode. However, this does not add anything essentially novel and only complicates notation.
} 
2.2. Pseudo continuity of the partial state. By pseudo-continuity, it will be understood that at the transition time $\tau_{i}$, the state $x_{i}$ in mode $i$ is mapped to state $x_{i+1}=S_{i} x_{i}$, where the switching matrices $\left\{S_{i}\right\}_{i=1}^{N}$ are fixed, with $S_{i} \in \mathbb{R}^{n_{i+1} \times n_{i}}$.

Note that if the cycle product $P_{1}^{N}=S_{N} S_{N-1} \cdots S_{2} S_{1}$ were nilpotent, any arbitrary initial state would be zeroed with $u \equiv 0$ in an infinitesimally short time simply by fast switching. We shall exclude this case by imposing that the ordered product $\prod_{i=1}^{N} S_{i}$ is not nilpotent.

A linear state transformation may be applied in each of the given modes. Indeed, letting for $T_{i} \in \mathrm{GL}_{n_{i}}(\mathbb{R}), T_{i} x_{i}=\bar{x}_{i}$, the effect on the switching matrices is

$$
\bar{S}_{i}=T_{i+1} S_{i} T_{i}^{-1},
$$

and transforms the ordered product $P_{1}^{N}$ by similarity, i.e.,

$$
\bar{P}_{1}^{N}=T_{1} P_{1}^{N} T_{1}^{-1}
$$

This induces a transformation $\mathbf{T}=\left\{T_{1}, \cdots, T_{N}\right\}$ on the discrete bundle. Such a transformation may be invoked to derive a canonical realization for the $M^{3} D$ system, for instance by requiring that $P_{1}^{N}$ is in the Jordan canonical form.

\section{Example}

Consider the case $N=2$, with $n_{1}=2, n_{2}=3$ and

$$
S_{1}=\left[\begin{array}{ll}
2 & 1 \\
1 & 1 \\
1 & 0
\end{array}\right] . \quad S_{2}=\left[\begin{array}{ccc}
1 & 2 & 3 \\
-1 & 2 & 0
\end{array}\right] \text {. }
$$

With

$$
T_{1}=\frac{1}{2}\left[\begin{array}{cc}
2 & 1 \\
0 & -1
\end{array}\right] . \quad T_{2}=\frac{1}{14}\left[\begin{array}{ccc}
1 & 6 & 6 \\
7 & -14 & 0 \\
6 & -6 & -6
\end{array}\right]
$$

we get

$$
\bar{S}_{1}=\left[\begin{array}{ll}
1 & 0 \\
0 & 1 \\
0 & 0
\end{array}\right] . \quad \bar{S}_{2}=\left[\begin{array}{lll}
7 & 0 & 0 \\
0 & 1 & 0
\end{array}\right]
$$

In general, one can make $S_{i}$ in the form $[I, 0]$ or $\left[\begin{array}{l}I \\ 0\end{array}\right]$ for $i=1, \cdots, N-1$, and $\bar{S}_{N}$ in Jordan canonical form (augmented with zeros). We note in passing that any cycle product $P_{k}^{N+k}$ will have the same nonzero eigenvalues. For this canonical form, the small dimensional state is embedded into a larger one, and a larger one is truncated to map to the smaller state space at the switching time. At the end of the cycle, the map $S_{N}$ in addition rescales the state components (in the simple case). 


\subsection{Examples of $M^{3} D$ Systems.}

2.3.1. Autonomous system (passive jumper). The first mode describes the jumper resting on the trampoline, so that the total mass is $M+m$, and the system has order two. If the velocity is sufficiently high, the jumper will be released from the platform, resulting in two uncoupled two-dimensional systems. See Figure 1.

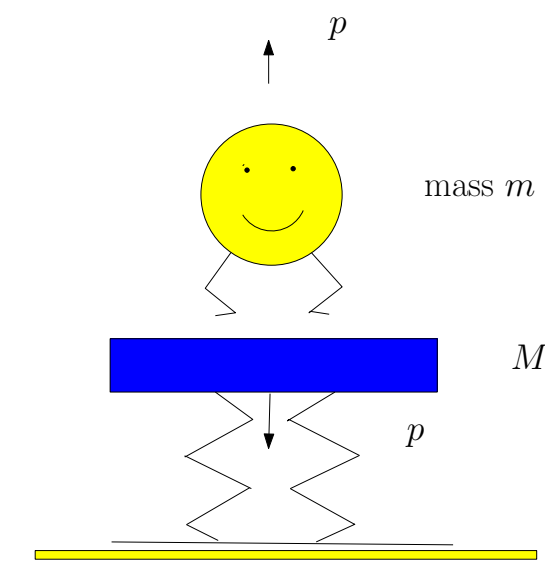

FIG. 1. The trampoline jumper $\underline{\text { Mode } 1}$

$$
\left\{\begin{array}{l}
\dot{x}=v \\
\dot{v}=-\frac{k}{M+m} x-\frac{g}{M+m}
\end{array}\right.
$$

Mode 2

$$
\begin{aligned}
& \left\{\begin{array}{l}
\dot{x}=v \\
\dot{v}=-\frac{k}{M} x-\frac{g}{M}
\end{array}\right. \\
& \left\{\begin{array}{l}
\dot{y}=u \\
\dot{u}=-\frac{g}{m}
\end{array}\right.
\end{aligned}
$$

Both modes occur if $x_{0}^{2}+\frac{v_{0}^{2}}{\omega_{0}^{2}} \geq\left(\frac{M g}{k}\right)^{2}$, where $\omega_{0}=\sqrt{k /(M+m) g}$ is the natural radial frequency when the jumper adheres to the platform.

Note that when the jumper is airborne, the platform oscillates with radial frequency $\sqrt{k / M g}$.

For precise initial conditions $\left(x_{0}, v_{0}\right)$ the hybrid system may exhibit a sustained oscillation. In the other cases the system is damped. For instance if the platform is pressed down and then released, the initial velocity is zero. Figure 2 shows for three different initial values of $x_{0}$, the motion of the jumper (dashed) and the platform (solid) as function of time.
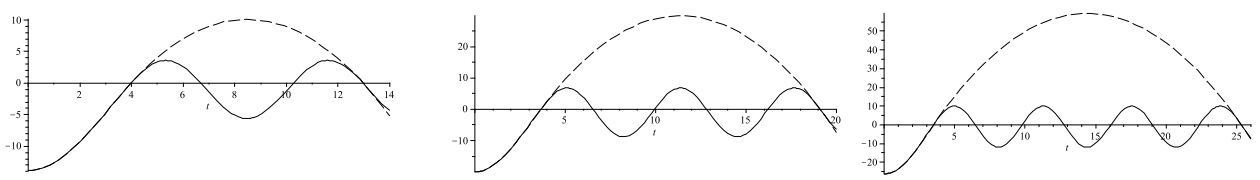

FIG. 2. Sustained oscillations for various initial conditions.

The active jumper uses impulsive control, and thus incurs the problem of timing the impulses, and choosing their magnitude, $p$. See Figure 1.

2.3.2. Switched Capacitor Circuit. Consider the simple switched capacitor circuit of Figure 3. The time constants are $\tau_{1}=R_{1} C_{1}, \tau_{2}=R_{2} C_{2}, \quad \tau_{0}=$ $\left(C_{1}+C_{2}\right) R_{1} R_{2} /\left(R_{1}+R_{2}\right)$. 


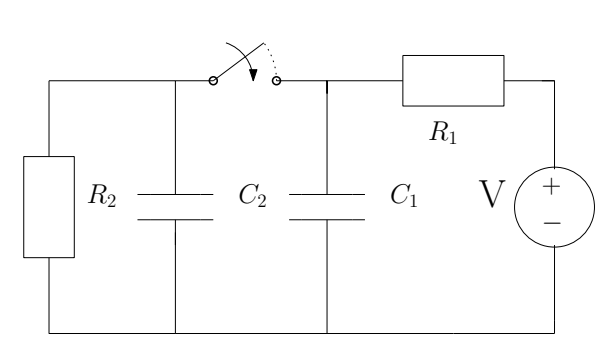

FIG. 3. Switched Circuit
Switch open: $\operatorname{dim}=2$

$$
\begin{aligned}
& \dot{q}_{1}+\frac{1}{\tau_{1}} q_{1}=\frac{V}{R_{1}} \\
& \dot{q}_{2}+\frac{1}{\tau_{2}} q_{2}=0
\end{aligned}
$$

Switch closed: $\operatorname{dim}=1$

$$
\dot{q}+\frac{1}{\tau_{0}} q=\frac{V}{R_{1}}
$$

The state transitions at the switches are given below:

$$
\begin{array}{ll}
\text { Open } \rightarrow \text { Closed: } & q=q_{1}+q_{2} \\
\text { Closed } \rightarrow \text { Open: } & {\left[\begin{array}{l}
q_{1} \\
q_{2}
\end{array}\right]=\frac{1}{C_{1}+C_{2}}\left[\begin{array}{l}
C_{1} \\
C_{2}
\end{array}\right] q .}
\end{array}
$$

2.3.3. Approximate models for nonlinear systems. Model reduction for a nonlinear system, evolving on $\mathbf{M}$ via balancing may lead to a system that has coordinate patches of differing dimensions. The reduced state space then has the structure of a CW complex [25]:

Indeed, in a balanced form, the canonical Gramian, $\Lambda: \mathbf{M} \rightarrow \mathbb{R}^{n}$, a positive definite matrix, characterizes the local input-to-state as well as the state-to-output properties (see [25]). $\Lambda$ defines a Riemannian metric on the tangent bundle TM. Let $\Lambda$ be approximated by $\widetilde{\Lambda}$ of rank $k \leq n$, in some subdomain of $\mathbf{M}$. If $x$ has the property $\operatorname{dim} \widetilde{\Lambda}(x)=k$, we say that $x$ lives in a patch of rank $k$. A smooth nonlinear system partitions $\mathbf{M}$ in patches of different ranks. The idea behind model reduction is to collapse the patch of rank $k$ to a $k$-dimensional cell. This gives a cell decomposition for the reduced state space. Note that in general, $\mathbf{M}$ will not be reduced to a single cell of some rank as in the LTI case! Model reduction in the nonlinear case is not uniform. A precise meaning of the reduced model must then be a hybrid system with modes of different dimension. Noninteresting dynamics may be forgotten in one patch, thus just keeping the interesting I-O part of the dynamics. Likewise, when crossing from a lower to a higher dimensional patch, the lost state components may be retrieved (by local observers). In this application, transitions are actually determined by switching manifolds.

Another instance stems from the approximation of a given system via piecewise linearization, having a different linear model (potentially of different dimension) in different subsets of the state space. 
2.3.4. Communication constrained control. In [6] an approximate stochastic model of different dimension for the observation and control mode for a remote system controlled via a one way communication link was considered, and an optimal switching policy established. The difference in dimensions stems here from the different reduction of the observer in both modes.

3. Randomly Switched Parameter Systems. In the remaining part of this paper it is assumed that the switching occurs at random times (a Poisson process) where perhaps the rate can be influenced. We first relate this problem to the socalled vibrational control problems, and review a simple scalar model whose random evolution is described by a hyperbolic PDE, known as the telegraphers equation. In the next subsection, we follow this outline to derive the backwards Kolmogorov equation for a randomly switched parameter system i.e., a multi-mode system with the same dimension in each mode. which will serve as a warmup for the general $P M^{3} D$ system in Section 4 .

3.1. Vibrational Control. In an influential paper [16], Meerkov introduced the idea of vibrational control. He proved that if $\dot{x}=A x$ is 'observable in principle', which means that there exists a $c$ such that the pair $(\mathrm{A}, \mathrm{c})$ is observable, then a necessary and sufficient condition for vibrational stabilizability is $\operatorname{Tr} A<0$. A vibrational control, $B(t)$, can then be found such that the system

$$
\dot{x}=[A+B(t)] x
$$

has the property $x(t) \rightarrow 0$ for any initial condition.

It was conjectured that a necessary and sufficient condition for the existence of a stationary random (matrix) process $W(t)$ such that

$$
\dot{x}=[A+W(t)] x
$$

is asymptotically stable with probability 1 , is also that $\operatorname{Tr} A<0$. This conjecture was soon proven to be a fact by Arnold, Crauel and Wihstutz [8]. In this section we consider a stochastic vibrational control of bang - bang type, governed by random switching of the modes in a $M^{3} D$ system.

In the remainder of this section we recall a simple model analyzed by Kac [14], which was the main inspiration for this work. The derivation is repeated here in order to provide some insight and to make this paper more self contained. The case of switching governed by a general Markov chain is considered in subsection 3. When the operation of the system alternates between two modes, more explicit descriptions can be given. This is taken up in section 4, and contains an application to stochastic stabilization. Preliminary work, leading to these results was reported in $[18,19]$. 
3.2. Kac's Randomly Switched Model. The following setup, taken from Kac [14], gives an elementary, yet insightful derivation of the backward Kolmogorov equation for a physically simple stochastic switched system. Consider a particle moving in one dimension, with speed $c$, but with direction switched at random times (Poisson arrival times, rate $\lambda)$. He analyzed the problem by first discretizing the interval $[0, t]$ into $N$ intervals of length $\Delta t$, and allowing switching only at times $n \Delta t$. Let $v_{n}$ be the speed in the $n$-th interval, then $v_{n+1}=\sigma_{n} v_{n}$, where $\sigma_{n}=-1$ if a switch occurs at $n \Delta t$, an event which happens with probability $\epsilon=\lambda \Delta t$; and $\sigma_{N}=1$ if no switch occurs. Clearly, the stochastic state equations are

$$
\begin{aligned}
v_{n+1} & =\sigma_{n} v_{n}, \\
x_{n+1} & =x_{n}+v_{n} \Delta t, \quad\left|v_{1}\right|=c
\end{aligned}
$$

These equations are readily iterated to

$$
v_{n}=\left(\sigma_{n-1} \cdots \sigma_{1}\right) v_{1},
$$

and for the displacement $(c=1 / \Delta t)$ :

$$
x_{n}=v_{1} \Delta t\left[1+\sigma_{1}+\sigma_{2} \sigma_{1}+\cdots+\left(\sigma_{n-1} \cdots \sigma_{1}\right)\right] \stackrel{\text { def }}{=} \frac{v_{1}}{c} y_{n} .
$$

Letting $x+y_{n}$ and $x-y_{n}$ be respectively the position of the particle starting at $x$ with initial velocity $v_{1}=c$ or $v_{1}=-c$, consider for any function $f$

$$
f_{n}^{ \pm}(x)=\mathbf{E} f\left(x \pm y_{n}\right)
$$

where the expectation is over the path, determined by $\sigma_{1}, \ldots, \sigma_{n}$. Taking the expectation first over $\sigma_{1}$, one derives

$$
f_{n}^{+}(x)=(1-\epsilon) f_{n-1}^{+}(x+c \Delta t)+\epsilon f_{n-1}^{-}(x+c \Delta t) .
$$

Upon taking limits for $\Delta t \rightarrow 0$, this yields finally:

$$
\frac{\partial f^{+}}{\partial t}=c \frac{\partial f^{+}}{\partial x}+\lambda\left(f^{-}-f^{+}\right) .
$$

Likewise, by symmetry,

$$
\frac{\partial f^{-}}{\partial t}=-c \frac{\partial f^{-}}{\partial x}+\lambda\left(f^{+}-f^{-}\right) .
$$

The equations (10) and (11) are known as the backward Kolmogorov equations. Introducing the combinations (symmetric and antisymmetric components)

$$
\begin{aligned}
& G=\frac{1}{2}\left(f^{+}+f^{-}\right) \\
& H=\frac{1}{2}\left(f^{+}-f^{-}\right) .
\end{aligned}
$$


Kac derived, after eliminating $H$, a hyperbolic equation known as the telegraphers equation.

$$
\frac{1}{c} \frac{\partial^{2} G}{\partial t^{2}}=c \frac{\partial^{2} G}{\partial x^{2}}-\frac{2 \lambda}{c} \frac{\partial G}{\partial t} .
$$

with the boundary conditions:

$$
\begin{aligned}
G(x, 0) & =f(x) \\
G_{t}(x, 0) & =0
\end{aligned}
$$

The latter being implied by $H(x, 0)=0$.

A number of interesting properties were discussed:

1. For $\lambda=0$, equation (14) reduces to the wave equation, which can be solved by the methods of characteristics. The solution is

$$
G(x, t)=\frac{1}{2}[f(x+c t)+f(x-c t)]
$$

2. For $\lambda \neq 0$, Kac expressed the displacement by a path integral

$$
\int_{0}^{t} v(\tau) d \tau=c \int_{0}^{t}(-1)^{N(\tau)} d \tau
$$

where $N(\tau)$ is the number of switches in the interval $[0, \tau)$. If $\left\{\tau_{k}\right\}$ are the Poisson arrival times, then,

$$
N(\tau)={ }^{\#}\left\{k \mid \tau_{k} \leq \tau\right\} .
$$

In terms of a stochastic time, $\mathcal{T}(\tau)=\int_{0}^{t}(-1)^{N(\tau)} d \tau$, the solution of the general equation (14) is represented by

$$
G(x, t)=\frac{1}{2} \mathbf{E}\{f(x+c \mathcal{T}(\tau))+f(x-c \mathcal{T}(\tau))]
$$

This form displays that the solution to the stochastic problem still has the structure of the underlying characteristics. In fact these ideas have been extended to a generalized characteristics method, encompassing not only the classical (deterministic) and Brownian methods of characteristics, but also for characteristic propagators of higher order order partial differential equations. See for instance $[9]$

3. In the limit $\lambda \rightarrow \infty, c \rightarrow \infty$, but $\lambda / c^{2}$ constant, the equation (14) reduces to

$$
\frac{\partial G}{\partial t}=\frac{c^{2}}{2 \lambda} \frac{\partial^{2} G}{\partial x^{2}}
$$

In the limit, the evolution is governed by the diffusion equation. 
3.2.1. General Markov Chains. The result of Kac has been extended by many other researchers to a general theory of random evolution, of which the monograph by Pinsky [17] gives an exciting overview. The theory has also found applications in quantum physics $[10,11,13]$.

Start with a randomly switched equi-dimensional systems of the form

$$
\frac{d x(t)}{d t}=F(t, x(t), \zeta(t)),
$$

where $\zeta(t)$, the state of a finite state Markov chain (hence piecewise constant) with state space $Z$, models a parameter process, or a randomly switching control. The process $x(t)$ is assumed to be continuous at the jump times of $\zeta(t)$. The combined process $(x(t), \zeta(t))^{\prime}$ is Markovian with state space $\mathbf{R}^{n} \times Z$. These processes are also referred to as piecewise deterministic processes. Next, the evolution operators $T_{t}^{z}$ are defined on a Banach space of space-time functions by

$$
T_{t}^{z} f(x, \tau)=f\left(\Phi_{z}(t+\tau, \tau, x), t+\tau\right),
$$

where

$$
\begin{aligned}
\frac{\partial}{\partial t} \Phi_{z}(t+\tau, \tau, x) & =F\left(t, \Phi_{z}(t+\tau, \tau, x), z\right) \\
\Phi_{z}(\tau, \tau, x) & =x .
\end{aligned}
$$

Thus $\Phi_{z}(t+\tau, \tau, x)$ solves the deterministic (for the mode parameterized by $z$ ) non autonomous ordinary differential equation with initial condition $x$ at time $\tau$. Said differently, $\Phi_{z}(t+\tau, \tau, x)$ is a parametrization (by $t$ ) of the trajectory of the system state passing through $x$ at time $\tau$ for mode $z$. Also note that $t$ represents the time lapse between the initial and the final time. For each $z$, the family $\left\{T_{t}^{z} \mid t>0\right\}$ is a contraction semigroup under the composition law. The semigroup property follows from the (assumed) uniqueness of the solution of the differential equation.

The general random evolution operator defines a vector valued Markov process on $\mathbf{R}^{n} \times Z$. This random evolution is studied by the expectation semigroup, defined by

$$
\tilde{T}_{t}^{z} f(x, \tau)=\mathbf{E}[f(x(t+\tau), t+\tau) \mid x(\tau)=x, \zeta(\tau)=z] .
$$

Its backward generator, $A$, is given by

$$
\begin{gathered}
{[A f(x, t)]_{z}=\frac{\partial f(x, t)_{z}}{\partial t}+F^{\prime}(t, x, z)\left[\nabla_{x} f(x, t)\right]^{\prime}+} \\
+\sum_{w \neq z} q(t, z, w)\left[f(x, t)_{w}-f(x, t)_{z}\right] .
\end{gathered}
$$


The prime denotes transposition, and $\nabla_{x} f=\frac{\partial f}{\partial x}$ is the row vector of partial derivatives of $f$ w.r.t. $x$. The second term is the crypto-deterministic term, stemming from the deterministic evolution in between the switches. The last term is due to the random switching, thus effectively mixing the underlying deterministic systems parameterized by $Z$. The matrix $q(t, \cdot, \cdot)$ is the generator of the Markov chain. The domain of $A$ consists of the set of functions $f(t, x, z)$ with, for each fixed $z \in Z$, continuous first order partial derivatives in $t$ and $x$.

3.3. Hyperbolic PDE for switched linear systems. For simplicity we limit our discussion to the case where all modes have linear time invariant autonomous state space representations

$$
\begin{aligned}
\dot{x}_{i} & =A_{i} x_{i} \\
y & =c_{i} x_{i} .
\end{aligned}
$$

Let, between switchings, $\zeta(t)$ indicate which mode is switched on at $t$. It will be shown that the evolution is described by (hyperbolic) PDE's, playing the same role as the (parabolic) backward Kolmogorov equation. The formal adjoint leads to the forward equation for the probabilities, which is again akin to the Fokker-Planck equation (See also [3]).

We first discuss the case where each mode has the same dimension. The complexity of the evolution equation is determined by the Lie algebra generated by the set $\left\{A_{i}\right\}$. It is shown that a parabolic equation is obtained as a special limiting case. Hence, the fast switching limit leads to diffusive behavior, in turn leading to a correction term for the drift. A system that is unstable on the average, may therefore be stabilizable by random switching between the different modes. This provides the stochastic extension of the philosophy of vibrational control introduced by Meerkov. The great benefit it that the 'control' proceeds in an unstructured fashion, i.e., without interfering in a deterministic way, hence also without the need of precise or even partial information on the states, as remarked in [8].

3.3.1. Equal Dimension Autonomous Case. Let $f$ be a differentiable function of $x$ and $t$, and consider the functions $u^{(i)}: \mathbb{R} \times \mathbb{R}^{n} \rightarrow \mathbb{R}$ by

$$
u^{(i)}(t, x)=u\left(t, x, z_{i}\right)=\mathbf{E}^{z_{i}} f\left[\Phi_{\zeta}(t, 0) x\right]
$$

thus defining the expectation semigroup for this case. $\left(\Phi_{\zeta}(t, 0) x\right.$ is the state at time $t$ if the initial condition at time zero was $x$.) This expectation is conditioned on the starting mode. Because of the time invariance, the starting time is taken at 0 . Since $Z$ is countable, denote $Z=\left\{z_{1}, \ldots, z_{N}\right\}$, where $N$ is possibly $\infty$. Let $A\left(\zeta_{t}=z_{i}\right)=A_{i}$, 
then the $u^{(i)}$, satisfy the backwards equation conditioned on the starting mode $z_{i}$ :

$$
\frac{\partial u^{(i)}}{\partial t}=\frac{\partial u^{(i)}}{\partial x} A_{i} x+\sum_{j \neq i} q_{i \rightarrow j}\left[u^{(j)}-u^{(i)}\right]
$$

where, for $j \neq i, q_{i \rightarrow j}$ is the rate of the transition $i \rightarrow j$ for the continuous time Markov jump process. The

$$
[q]_{i \rightarrow j}=\left.\frac{d}{d \tau} \operatorname{Pr}_{t, \tau}(i \rightarrow j)\right|_{\tau \rightarrow t_{+}},
$$

are also known as the infinitesimal parameters. These transition rates define a transition probability rate matrix $Q$, with for $i \neq j: Q_{i j}=q_{i \rightarrow j}$ and $Q_{i i}=-\sum_{j \neq i} Q i j$ [12]. The elements in the rows of $Q$ sum to 0 .

In vector form, setting $u^{\prime}=\left[u^{(1)}, \ldots, u^{(N)}\right]$, the coupled backward equations are

$$
\frac{\partial u}{\partial t}=\left[\begin{array}{ccc}
x^{\prime} A_{1}^{\prime}\left(\frac{\partial}{\partial x}\right)^{\prime} & & \\
& \ddots & \\
& & x^{\prime} A_{N}^{\prime}\left(\frac{\partial}{\partial x}\right)^{\prime}
\end{array}\right] u+Q u .
$$

Let us simplify the notation by defining the symbols: $\partial_{i}=x^{\prime} A_{i}^{\prime}\left(\frac{\partial}{\partial x}\right)^{\prime}$ and $\partial=\frac{\partial}{\partial t}$. Then the system (33) is compactly written as the vector PDE

$$
\partial u=\operatorname{diag}\left(\partial_{i}\right) u+Q u
$$

This system of equations can be rewritten in terms of symmetrical components, thus generalizing the sum and difference considered by Kac. Using the discrete Fourier transform, introduce the variables

$$
\hat{u}^{m}=\sum_{k} \mathcal{F}_{m k} u^{(k)}
$$

where

$$
\mathcal{F}_{m k}=\frac{1}{N} e^{j 2 \pi \frac{(m-1)(k-1)}{N}}
$$

is the $m k$ entry of the (discrete) Fourier transform matrix. In view of the row sum property of the stochastic matrix, the equation (34) transforms to a symmetric component form

$$
\partial \hat{u}=\Delta \hat{u}+\widehat{Q} \hat{u}
$$

where now $\widehat{Q}_{11}=0$.

An interesting class of multi mode systems is the class of asynchronously cyclic systems. It is assumed that none of the modes in $Z$ are absorbing, and that transitions have a cyclic order. This class is the stochastic generalization of the class 
of periodic systems. It is known that linear periodic controllers enhance the closed loop characteristics of deterministic time-invariant plants. Topics such as gain margin augmentation, blocking zero removal, and the simultaneous stabilization of a finite family of linear time-invariant plants have been addressed in the control literature. Similar questions can be asked for the class of cyclic multi mode systems.

Consider an asynchronously cyclic system with $N$ modes, each having the same average sojourn time (equal to $\frac{1}{\lambda}$, consistent with an exponential distribution). For this system, the transition probability rate matrix is given by $\lambda(-I+C)$, where $C$ is the circulant matrix

$$
C=\left[\begin{array}{cccc}
0 & 1 & & \\
\vdots & \ddots & \ddots & \\
\vdots & & \ddots & 1 \\
1 & \cdots & \cdots & 0
\end{array}\right]
$$

Pre multiplication of the hyperbolic system of equations by $\mathcal{F}$, and postmultiplication by $\mathcal{F}^{*}$ yields after some algebra, the symmetric component form

(37), with

$$
(\Delta)_{k l}=\sum_{\alpha} \partial_{\alpha} \mu^{\alpha(k-l)} \quad ; \quad \mu=e^{\frac{j 2 \pi}{N}}
$$

and

$$
\widehat{Q}=\lambda N\left[\begin{array}{llll}
0 & & & \\
& \bar{\mu}-1 & & \\
& & \ddots & \\
& & & \bar{\mu}^{N}-1
\end{array}\right]
$$

The differential operator matrix $\Delta$ is Toeplitz (in fact circulant), and the mixing operator $\widehat{Q}$ is diagonal. In general, the symmetric components yield complex coupled equations, except for the case $N=2$.

Example: The three-cyclic second order multi mode system with

$$
A_{1}=-3 I \quad ; \quad A_{2}=\left[\begin{array}{cc}
0 & \sqrt{3} \\
-\sqrt{3} & 0
\end{array}\right]=-A_{3} \quad ;
$$

is completely commutative $\left(\left[A_{1}, A_{2}\right]=\left[A_{2}, A_{3}\right]=\left[A_{3}, A_{1}\right]=0\right)$, and has discrete Fourier transform $\left(\bar{\mu}=\mu^{-1}=\mu^{2}\right)$

$$
\hat{\mathcal{A}}(\mu)=\left[\begin{array}{cc}
-1 & \frac{\mu-\mu^{2}}{\sqrt{3}} \\
\frac{\bar{\mu}-\mu}{\sqrt{3}} & -1
\end{array}\right] \text {. }
$$


Consequently, its symmetric components are

$$
\begin{gathered}
\hat{A}_{1}=\hat{\mathcal{A}}(1)=-I ; \quad \hat{A}_{2}=\hat{\mathcal{A}}(\mu)=\left[\begin{array}{cc}
-1 & j \\
-j & -1
\end{array}\right] ; \\
\hat{A}_{3}=\hat{\mathcal{A}}\left(\mu^{2}\right)=\left[\begin{array}{cc}
-1 & -j \\
j & -1
\end{array}\right] .
\end{gathered}
$$

In the sequel, we will also need the commutator for the differential operators $\partial_{i}=x^{\prime} A_{i}^{\prime}\left(\frac{\partial}{\partial x}\right)^{\prime}$, namely

$$
\begin{aligned}
{\left[\partial_{i}, \partial_{j}\right] } & =\left[x^{\prime} A_{i}^{\prime}\left(\frac{\partial}{\partial x}\right)^{\prime}, x^{\prime} A_{j}^{\prime}\left(\frac{\partial}{\partial x}\right)^{\prime}\right] \\
& =x^{\prime}\left[A_{j}, A_{i}\right]^{\prime}\left(\frac{\partial}{\partial x}\right)^{\prime} \\
& \stackrel{\text { def }}{=} \partial_{[j, i]} .
\end{aligned}
$$

The notation indicates that the Lie-product operator corresponds again to a linear vector field.

3.4. Alternating Systems. In this section we consider systems having only two modes $(N=2)$, which we shall denote by + and - , corresponding to $\dot{x}=A_{+} x$, and $\dot{x}=A_{-} x$. The "time-average" system is $\dot{x}=A_{0} x$, with $A_{0}=\frac{1}{2}\left[A_{+}+A_{-}\right]$. We shall define the "excursion" by $\Omega=\frac{1}{2}\left[A_{+}-A_{-}\right]$.

Then $\hat{u}^{\prime}=\left[\frac{1}{2}\left(u_{+}+u_{-}\right), \frac{1}{2}\left(u_{+}-u_{-}\right)\right]^{\prime}=[G, H]^{\prime}$. Similarly, denote $\partial_{1}=\partial_{+}, \partial_{2}=\partial_{-}$, and define the symmetric operators $\partial_{0}=\frac{1}{2}\left(\partial_{+}+\partial_{-}\right)$, and $\partial_{\omega}=\frac{1}{2}\left(\partial_{+}-\partial_{-}\right)$. Finally set $p=\partial-\partial_{0}$.

We first derive the PDE for $G$ (the true average over both initial modes) and its initial conditions. Then we show that the behavior in the limit for fast switching is modeled by a parabolic PDE, associated with a linear Itô system. The stability properties of this limit system, and therefore also the long term behavior of the switched system is investigated via this Itô representation. We close this section with some illustrative examples.

3.4.1. Hyperbolic Backward Equation. The system of PDE's is given in terms of its symmetric components by

$$
\left[\begin{array}{c}
p \\
\partial_{\omega}
\end{array}\right] H=\left[\begin{array}{c}
\partial_{\omega} \\
p
\end{array}\right] G+\left[\begin{array}{c}
-2 \lambda \\
0
\end{array}\right] H
$$

To obtain a higher order partial differential equation in $G$ only, $H$ needs to be eliminated. Assume that, for some $p_{k}, q_{k}$, the following relation holds:

$$
\left[\begin{array}{c}
p \\
q_{k}
\end{array}\right] H=\left[\begin{array}{c}
\partial_{\omega} \\
p_{k}
\end{array}\right] G+\left[\begin{array}{c}
-2 \lambda \\
0
\end{array}\right] H .
$$


Then, operating on the left with the $1 \times 2$ operator $\left[-q_{k} p\right]$, not to be confused with a Lie product (Lie bracket), one obtains

$$
\left[p, q_{k}\right] H=\left(-q_{k} \partial_{\omega}-p p_{k}+2 \lambda p_{k}\right) G
$$

This is put in the form

$$
q_{k+1} H=p_{k+1} G,
$$

thus defining the recursions:

$$
\begin{aligned}
q_{k+1} & =\left[p, q_{k}\right] \\
p_{k+1} & =-q_{k} q+p p_{k}+2 \lambda p_{k} .
\end{aligned}
$$

The 'initial conditions' for this recursion are: $q_{0}=q=\partial_{\omega}$, and $p_{0}=p=\partial-\partial_{0}$. Invoking the 'ad'-notation for repeated Lie brackets, we have

$$
q_{k}=\operatorname{ad}_{p}^{k} q \stackrel{\text { def }}{=}\left[p,\left[p, \ldots\left[p, q_{0}\right] \ldots\right]\right], \quad(k \text { brackets }) .
$$

Combine the set of equations in matrix form, we obtain

$$
\left[\begin{array}{c}
p_{0} \\
q_{0} \\
q_{1} \\
q_{2} \\
\vdots \\
q_{k}
\end{array}\right] H=\left[\begin{array}{c}
q_{0} \\
p_{0} \\
p_{1} \\
p_{2} \\
\vdots \\
p_{k}
\end{array}\right] G+\left[\begin{array}{c}
-2 \lambda \\
0 \\
0 \\
0 \\
\vdots \\
0
\end{array}\right] H .
$$

Note that $\operatorname{ad}_{p}^{k} q=(-1)^{k} \operatorname{ad}_{\partial_{0}}^{k} \partial_{\omega}$, so that the sequence $\left\{\operatorname{ad}_{p}^{k} q\right\}$ is completely determined by the sequence of matrix commutators $\left\{\operatorname{ad}_{A_{+}}^{k} A_{-}\right\}$, or $\left\{\operatorname{ad}_{A_{0}}^{k} \Omega\right\}$. Hence there exists an integer $m<n^{2}+1$ and scalars $\alpha_{1}, \ldots, \alpha_{m}$ such that

$$
\operatorname{ad}_{p}^{m} q=-\sum_{i=1}^{m} \alpha_{i} \operatorname{ad}_{p}^{m-i} q
$$

In turn, this yields, with $\alpha_{0}=1$, the higher order PDE

$$
\sum_{i=0}^{m} \alpha_{i} p_{m-i} G=0
$$

with initial data $G(0, x)=\phi(x), H(0, x)=0$. Note also that the relation (41) implies that $\operatorname{ad}_{p}^{k} q=(-1)^{k} \operatorname{ad}_{\partial_{0}}^{k} \partial_{\omega}=\operatorname{ad}_{p}^{k} \partial_{\omega}$, are differential operators of first order in $x$. On the other hand, the $p_{k}$ are operators of order $k+1$ in both $x$ and time $\tau$. So the PDE we obtained is of higher order, in fact $m+1$, and more derivatives of $G$ need to be specified in this Cauchy problem. This information is already present in the 
data. Indeed, since at $t=0, H \equiv 0$, we get also $q_{k} H \equiv 0$ (which is easily shown by induction), leading to $p_{k} G=0$ at $t=0$.

To proceed further, rewrite the basic coupled PDE's in the following form:

$$
\partial\left[\begin{array}{c}
G \\
H
\end{array}\right]=\left[\begin{array}{cc}
\partial_{0} & \partial_{\omega} \\
\partial_{\omega} & \partial_{0}-2 \lambda
\end{array}\right]\left[\begin{array}{c}
G \\
H
\end{array}\right] .
$$

By repeated differentiation with respect to $t$, we get, by virtue of the commutation of $\partial$ with both of $\partial_{0}$ and $\partial_{\omega}$,

$$
\partial^{k}\left[\begin{array}{l}
G \\
H
\end{array}\right]=\left[\begin{array}{cc}
\partial_{0} & \partial_{\omega} \\
\partial_{\omega} & \partial_{0}-2 \lambda
\end{array}\right]^{k}\left[\begin{array}{c}
G \\
H
\end{array}\right] .
$$

Evaluating the expression at $t=0$, and keeping in mind that at $t=0, G(x, 0) \equiv \phi(x)$ and $H(x, 0) \equiv 0$, then the initial conditions follow from

$$
\left.\partial^{k} G\right|_{t=0}=\left[\begin{array}{ll}
1 & 0
\end{array}\right]\left[\begin{array}{cc}
\partial_{0} & \partial_{\omega} \\
\partial_{\omega} & \partial_{0}-2 \lambda
\end{array}\right]^{k}\left[\begin{array}{l}
\phi \\
0
\end{array}\right] .
$$

This completely specifies the Cauchy problem.

3.4.2. Limit Parabolic System. Next, let us investigate the limit system for very fast switching, and large excursions. Recall first that for an Itô process, $x(t)$, modeled by

$$
d x=f(x) d t+g(x) d w(t)
$$

where $w(t)$ is an $m$-dimensional standard Brownian motion, and where $f$ and $g$ satisfy the usual regularity conditions, there is an associated diffusion process. For any $u(x, t)$, twice differentiable with respect to the components of $x$ and once with respect to $t$, this associated diffusion satisfies Kolmogorov's backward equation

$$
\frac{\partial}{\partial t} u(x, t)=\nabla u(x, t) \cdot f(x, t)+\frac{1}{2} \operatorname{Tr} g g^{\prime} \operatorname{Hess} u(x, t) .
$$

Renormalize for the switching system, the large excursions by setting $\Omega=\omega \Omega_{0}$, where $\Omega_{0}$ is some normalized excursion, and $\omega$ a "strength"-parameter. Consider also a high switching rate, $\lambda$. It is shown next that the limit for $\lambda \rightarrow \infty$ and $\omega \rightarrow \infty$, but such that $\sigma^{2}=\frac{\omega^{2}}{2 \lambda}$ remains constant, reduces the hyperbolic PDE to a parabolic PDE of the form (56). Consequently, the sample trajectories can be represented by the solution of an Itô system, which is important from the point of view of the stability analysis.

TheOREM 1. If $\lambda \rightarrow \infty$ and $\omega \rightarrow \infty$, but with $\sigma^{2}=\frac{\omega^{2}}{2 \lambda}$ kept constant, then the evolution of the limiting alternating system is governed by the parabolic PDE

$$
\left(\partial-\partial_{0}-\sigma^{2}{\overline{\partial_{\omega}}}^{2}\right) G=0
$$


where now $\overline{\partial_{\omega}}=x^{\prime} \Omega_{0}^{\prime}\left[\frac{\partial}{\partial x}^{\prime}\right]$. This holds, independent of the commutativity properties (of $A_{0}$ and $\Omega_{0}$ ).

Note that by expanding the symbols,

$$
\begin{aligned}
{\overline{\partial_{\omega}}}^{2} & =x^{\prime} \Omega_{0}^{\prime}\left(\frac{\partial}{\partial x}\right)^{\prime} x^{\prime} \Omega_{0}^{\prime}\left(\frac{\partial}{\partial x}\right)^{\prime} \\
& =x^{\prime} \Omega_{0}^{\prime 2}\left(\frac{\partial}{\partial x}\right)^{\prime}+\operatorname{Tr}\left[\Omega_{0} x x^{\prime} \Omega_{0}^{\prime}\left(\frac{\partial^{2}}{\partial x \partial x^{\prime}}\right)\right],
\end{aligned}
$$

so that in terms of the original problem data, the parabolic PDE (57) is

$$
\frac{\partial G}{\partial t}=\frac{\partial G}{\partial x}\left(A_{0}+\sigma^{2} \Omega_{0}^{2}\right) x+\sigma^{2} \operatorname{Tr}\left(\Omega_{0} x x^{\prime} \Omega_{0}^{\prime} \operatorname{Hess} G\right),
$$

where 'Hess $G$ ' denotes the Hessian matrix of $G$.

Proof. First observe that the recursions (46) and (47) for the differential operators give

$$
p_{k+1}=-\left[\operatorname{ad}_{p}^{k} \overline{\partial_{\omega}}\right] \overline{\partial_{\omega}} \omega^{2}+p p_{k}+2 \lambda p_{k}
$$

Dividing by $(2 \lambda)^{k+1}$ we get

$$
\frac{p_{k+1}}{(2 \lambda)^{k+1}}=-\left[\operatorname{ad}_{p}^{k} \overline{\partial_{\omega}}\right] \overline{\partial_{\omega}} \frac{\omega^{2}}{2 \lambda} \frac{1}{(2 \lambda)^{k}}+\frac{p}{2 \lambda} \frac{p_{k}}{(2 \lambda)^{k}}+\frac{p_{k}}{(2 \lambda)^{k}} .
$$

Letting for $k=1,2, \ldots$

$$
\pi_{k}=\frac{p_{k}}{(2 \lambda)^{k}}
$$

then we get

$$
\pi_{k+1}=-\frac{1}{(2 \lambda)^{k}} \sigma^{2}\left[\operatorname{ad}_{p}^{k} \overline{\partial_{\omega}}\right] \overline{\partial_{\omega}}+\frac{\partial-\partial_{0}}{2 \lambda} \pi_{k}+\pi_{k} \longrightarrow \pi_{k}
$$

as $\lambda \rightarrow \infty$, but keeping $\frac{\omega^{2}}{2 \lambda}=\sigma^{2}$. Thus, for $k \geq 1$, we find $\pi_{k+1}=\pi_{k}$, while by direct calculation, (47) yields $\pi_{1} \rightarrow \partial-\partial_{0}-\sigma^{2}{\overline{\partial_{\omega}}}^{2}$.

Thus in all cases, i.e., whether commuting or not, we find the same diffusion limit system (60). In the sequel, we shall characterize the limit system by the triple $\left(A_{0}, \Omega_{0}, \sigma^{2}\right)$.

3.4.3. Stability of the Limit System. We first recall some general properties. For the appropriate definitions of stability we refer to the literature $[7,15]$. See also J.L. Willems for some explicit stability criteria [20,21]. A necessary (resp. sufficient) condition for exponential stability in mean square of the Itô system

$$
d x=A x d t+B x d w(t)
$$


is that for every (for some) symmetric positive definite matrices $R$, there exist a positive definite symmetric solution $P$ of the generalized Lyapunov equation

$$
P A+A^{\prime} P+B^{\prime} P B+R=0 .
$$

Exponential stability in mean square implies weak stochastic stability, and for autonomous systems, is implied by stochastic stability [7, p.196]. By a theorem of Khasminskii, weak stochastic stability and (strong) stochastic stability are equivalent for linear time invariant systems [15, p. 245]. If the equilibrium of a linear equation is asymptotically stable, it is automatically globally asymptotically stable (G.A.S.). If $A$ and $B$ are commuting, more can be said: Indeed, the solution to the SDE is [7, p. 144]

$$
x(t)=\exp \left[\left(A-\frac{1}{2} B^{2}\right) t+B w(t)\right] x_{0}
$$

which is easily verified with the Itô differential rule. Since $\frac{w(t)}{t} \rightarrow 0$ a.s., this verifies that global asymptotic stability will hold if $A-\frac{1}{2} B^{2}$ has all its eigenvalues in the left half plane.

These remarks lead directly to the following:

TheOREM 2. If symmetric positive definite matrices $P$ and $Q$ exist, such that

$$
P\left(A_{0}+q \Omega_{0}^{2}\right)+\left(A_{0}+q \Omega_{0}^{2}\right)^{\prime} P+2 q \Omega_{0}^{\prime} P \Omega_{0}+Q=0 .
$$

then the alternating system $\left(A_{0}, \Omega_{0}, q\right)$ is globally asymptotically stable.

Proof. The diffusion equation (60) is equivalent to the Itô representation

$$
d x=\left(A_{0}+q \Omega_{0}^{2}\right) x d t+\sqrt{2 q} \Omega_{0} x d w(t) .
$$

Thus we shall have global asymptotic stability if the condition (68) holds.

Corollary. If the modal matrices $A_{+}$and $A_{-}$commute, then the limit of the randomly switched system is G.A.S. iff the averaged system $A_{0}=\frac{1}{2}\left[A_{+}+A_{-}\right]$is asymptotically stable.

Proof. Under the given condition, $A_{0}$ and $\Omega_{0}$ commute. Then, the G.A.S. condition is by (67) the asymptotic stability of

$$
\left(A_{0}+q \Omega_{0}^{2}\right)-\frac{1}{2}(2 q) \Omega_{0}^{2}=A_{0} .
$$




\subsubsection{Examples.}

1. Scalar system: $\dot{x}=\left(a_{0} \pm \omega\right) x$. One finds $q_{1}=\left[p_{0}, q_{0}\right]=0$. Hence the resulting $\mathrm{PDE}$ is $p_{1} G=0$ or,

$$
\left[\left(\frac{\partial}{\partial \tau}-a_{0} x \frac{\partial}{\partial x}\right)^{2}-\left(\omega x \frac{\partial}{\partial x}\right)^{2}+2 \lambda\left(\frac{\partial}{\partial \tau}-a_{0} x \frac{\partial}{\partial x}\right)\right] G=0 .
$$

The limit system, with $\frac{\omega^{2}}{2 \lambda}=\sigma^{2}$ is

$$
\frac{\partial G}{\partial \tau}=\left(a_{0}+\sigma^{2}\right) x \frac{\partial G}{\partial x}+\sigma^{2} x^{2} \frac{\partial^{2} G}{\partial x^{2}} .
$$

This limit system satisfies the Itô equation:

$$
d x=\left(a_{0}+\sigma^{2}\right) x d t+\sqrt{2 \sigma^{2}} x d w(t),
$$

and is G.A.S. if $a_{0}<0$.

2. Consider the second order system

$$
\dot{x}=a_{0} x d t \pm\left[\begin{array}{cc}
\sigma & \omega \\
-\omega & \sigma
\end{array}\right] x d w(t)
$$

Here obviously, the matrices $A_{0}$ and $\Omega$ (or $A_{+}$and $A_{-}$commute). Introducing $\rho=\sigma / \omega$ the two modes of the system are

$$
\begin{aligned}
& A_{+}=\left[\begin{array}{cc}
a_{0}+\omega \rho & \omega \\
-\omega & a_{0}+\omega \rho
\end{array}\right] \\
& A_{-}=\left[\begin{array}{cc}
a_{0}-\omega \rho & -\omega \\
\omega & a_{0}-\omega \rho
\end{array}\right] .
\end{aligned}
$$

Both modes have a complex conjugate pole pair $(\omega \neq 0)$ and hence are oscillatory. The limit diffusion $\left(\frac{\omega^{2}}{2 \lambda}=\sigma^{2}\right)$ is associated with the Itô process

$$
d x=\left(a_{0} I+\sigma^{2} \Omega^{2}\right) x d t+\sqrt{2 \sigma^{2}} \Omega x d w(t) .
$$

It does not depend on the parameter $\rho$. We have G.A.S. if $a_{0}<0$, i.e., if the averaged system is G.A.S. Note that one mode, $A_{+}$or $A_{-}$, may be unstable, as long as the average is stable.

3. The second order system

$$
\dot{x}=-\sigma x d t \pm\left[\begin{array}{ll}
2 \sigma & \\
& -2 \sigma
\end{array}\right] x d w(t)
$$

has two unstable modes.

$$
A_{+}=\left[\begin{array}{ll}
\sigma & \\
& -3 \sigma
\end{array}\right] \quad ; \quad A_{-}=\left[\begin{array}{ll}
-3 \sigma & \\
& \sigma
\end{array}\right] .
$$

Both have eigenvalues $\{\sigma,-3 \sigma\}$. The matrices $A_{0}$ and $\Omega$ (or $A_{+}$and $A_{-}$) commute, hence by the Corollary, the system is G.A.S. if $\sigma>0$. 
4. Consider the noncommuting second order system with

$$
A_{0}=\left[\begin{array}{ll}
\lambda_{1} & \\
& \lambda_{2}
\end{array}\right] \quad ; \quad \Omega=\left[\begin{array}{cc}
0 & \omega \\
0 & 0
\end{array}\right],
$$

where $\lambda_{1} \neq \lambda_{2}$. The Lie brackets are

$$
\begin{gathered}
{\left[A_{0}, \Omega\right]=\left(\lambda_{1}-\lambda_{2}\right) \Omega,} \\
{\left[A_{0},\left[A_{0}, \Omega\right]\right]=\left(\lambda_{1}-\lambda_{2}\right)^{2} \Omega,} \\
{\left[\Omega,\left[A_{0}, \Omega\right]\right]=0 .}
\end{gathered}
$$

Hence $q_{1}=\left[p_{0}, q_{0}\right]=-\left[\partial_{0}, \partial_{\omega}\right]=\left(\lambda_{1}-\lambda_{2}\right) \partial_{\omega}$, from which $p_{1} G=\left(\lambda_{2}-\right.$ $\left.\lambda_{1}\right) p_{0} G$, i.e.,

$$
\begin{aligned}
& \left(\frac{\partial}{\partial \tau}-\lambda_{1} x_{1} \frac{\partial}{\partial x_{1}}-\lambda_{2} x_{2} \frac{\partial}{\partial x_{2}}\right)^{2}-\omega x_{2} \frac{\partial}{\partial x_{1}} \omega x_{2} \frac{\partial}{\partial x_{1}}+ \\
& \quad+2 \lambda\left(\frac{\partial}{\partial \tau}-\lambda_{1} x_{1} \frac{\partial}{\partial x_{1}}-\lambda_{2} x_{2} \frac{\partial}{\partial x_{2}}\right) \\
& =\left(\lambda_{1}-\lambda_{2}\right)\left(\frac{\partial}{\partial \tau}-\lambda_{1} x_{1} \frac{\partial}{\partial x_{1}}-\lambda_{2} x_{2} \frac{\partial}{\partial x_{2}}\right) .
\end{aligned}
$$

The limit system, with $\frac{\omega^{2}}{2 \lambda}=\sigma^{2}$, is

$$
\frac{\partial G}{\partial \tau}=\left[\lambda_{1} x_{1} \frac{\partial G}{\partial x_{1}}+\lambda_{2} x_{2} \frac{\partial G}{\partial x_{2}}\right]+\sigma^{2} x_{2}^{2} \frac{\partial^{2} G}{\partial x_{1}^{2}}
$$

which is in fact the same limit as one would have obtained under the commutation assumption. The corresponding Itô system is

$$
\begin{aligned}
{\left[\begin{array}{l}
d x_{1} \\
d x_{2}
\end{array}\right]=} & {\left[\begin{array}{ll}
\lambda_{1} & \\
& \lambda_{2}
\end{array}\right]\left[\begin{array}{l}
x_{1} \\
x_{2}
\end{array}\right] d t+} \\
& +\sqrt{2 \sigma^{2}}\left[\begin{array}{ll}
0 & 1 \\
0 & 0
\end{array}\right]\left[\begin{array}{l}
x_{1} \\
x_{2}
\end{array}\right] d w(t) .
\end{aligned}
$$

G.A.S. holds if there exist positive $Q_{11}, Q_{22}$ such that the following equations have positive solutions for $P_{11}$ and $P_{22}$ :

$$
\begin{aligned}
2 \lambda_{1} P_{11}+2 q P_{22}+Q_{11} & =0 \\
2 \lambda_{2} P_{22}+Q_{22} & =0
\end{aligned}
$$

Obviously, the above conditions can only hold if both $\lambda_{1}<0, \lambda_{2}<0$. 
5. The final example may be the most interesting one. It illustrates that in the noncommutative case, G.A.S. may hold, even if the time-averaged system is unstable! Consider the following (noncommuting) example

$$
A_{0}=\left[\begin{array}{ll}
\lambda_{1} & \\
& \lambda_{2}
\end{array}\right] \quad ; \quad \Omega_{0}=\left[\begin{array}{cc} 
& 1 \\
-1 &
\end{array}\right] .
$$

The generalized Lyapunov equation leads to the system

$$
\begin{aligned}
2\left(\lambda_{1}-\sigma^{2}\right) p_{11}+2 \sigma^{2} p_{22}+r_{11} & =0 \\
2\left(\lambda_{2}-\sigma^{2}\right) p_{22}+2 \sigma^{2} p_{11}+r_{22} & =0 \\
2\left(\frac{\lambda_{1}+\lambda_{2}}{2}-\sigma^{2}\right) p_{12}-2 \sigma^{2} p_{12}+r_{12} & =0
\end{aligned}
$$

Its solution is

$$
\begin{aligned}
& p_{11}=\frac{1}{2 \Delta}\left[\sigma^{2}\left(r_{11}+r_{22}\right)-\lambda_{2} r_{11}\right] \\
& p_{22}=\frac{1}{2 \Delta}\left[\sigma^{2}\left(r_{11}+r_{22}\right)-\lambda_{1} r_{22}\right] \\
& p_{12}=\frac{r_{12}}{4 \sigma^{2}-\left(\lambda_{1}+\lambda_{2}\right)}
\end{aligned}
$$

where

$$
\Delta=\lambda_{1} \lambda_{2}-\left(\lambda_{1}+\lambda_{2}\right) \sigma^{2}=\left(\lambda_{1}-\sigma^{2}\right)\left(\lambda_{2}-\sigma^{2}\right)-\sigma^{4} .
$$

If $\Delta<0$, a positive definite solution to the Lyapunov equation does not exist. But if $\Delta>0$, then the additional conditions are $\sigma^{2}-\lambda_{i}>0$. In the $\left(\lambda_{1}, \lambda_{2}\right)$-parameter plane, we get a stability region bounded by the branch of the hyperbola with top at the origin and vertical and horizontal asymptotes respectively through $\left(\sigma^{2}, 0\right)$ and $\left(0, \sigma^{2}\right)$. For $\sigma=0$, the third quadrant is the domain of stability, but for increasing $\sigma$ this domain is enlarged. Note however that at least one of the eigenvalues of $A_{0}$ needs to be negative. As $\sigma \rightarrow \infty$, all pairs below the line $\lambda_{2}=-\lambda_{1}$ are stabilizable. (Figure 4). Thus it follows that if the average eigenvalue of the system is negative, i.e., $\operatorname{Tr} A_{0}<0$, stabilizability is possible with fast switching, even though the averaged system $\left(A_{0}\right)$ may not be stable.

4. Kolmogorov PDE for $P M^{3} D$ Systems. In this section we consider the Poisson switching for the $M^{3} D$ model described in Section 2. As the state space is a discrete bundle in this case, we use a new (albeit somewhat redundant) notation. Represent the state by $\left(\zeta ; x_{1}, x_{2}, \ldots, x_{N}\right) \in \mathbf{X}=\mathbf{\Xi} \times\left(\mathbb{R}^{\mathbf{n}_{\mathbf{1}}} \oplus \cdots \oplus \mathbb{R}^{\mathbf{n}_{\mathbf{N}}}\right)$ equipped with the equivalence: $\forall i=1, \ldots, N ; \zeta=i$ implies

$$
\left(\zeta ; x_{1}, \cdots, x_{i-1}, x_{i}, x_{i+1} \cdots x_{N}\right) \sim\left(\zeta ; x_{1}^{\prime}, \cdots, x_{i-1}^{\prime}, x_{i}, x_{i+1}^{\prime} \cdots x_{N}^{\prime}\right),
$$




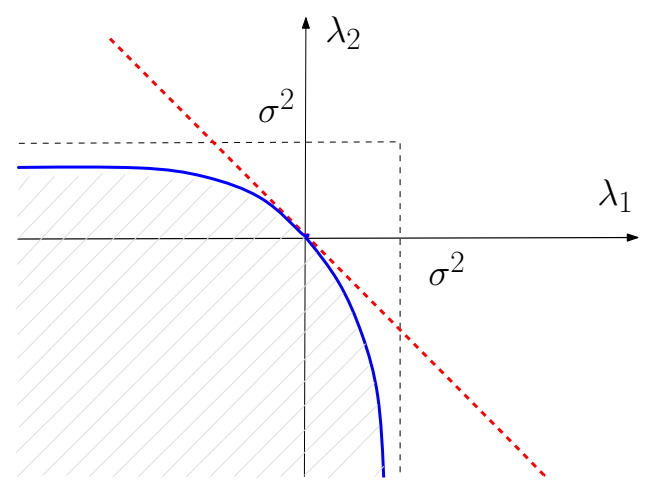

FIG. 4. Stability region in $\left(\lambda_{1}, \lambda_{2}\right)$-plane.

for all $\left(x_{1}^{\prime}, \ldots, x_{i-1}^{\prime}, x_{i+1}^{\prime}, \ldots, x_{N}^{\prime}\right) \in\left(\mathbb{R}^{n_{1}} \oplus \cdots \mathbb{R}^{n_{i-1}} \oplus \mathbb{R}^{n_{i+1}} \oplus \mathbb{R}^{n_{N}}\right)$. This means that only the $n_{i}$-dimensional state component $x_{i}$ is relevant when $\zeta=i$.

Let $f$ be an arbitrary differentiable function defined on this state space $\mathbf{X}$, thus satisfying, when $\zeta=i$

$$
f(i, \mathbf{x})=f\left(i, \mathbf{x}^{\prime}\right) \text { with } x_{i}^{\prime}=x_{i}
$$

With some abuse of notation, collapse this notation again to $f\left(i, x_{i}\right)$, and consider the path functionals

$$
F^{(i)}(\zeta, \mathbf{x}, t)=F\left(t, i, x_{i}\right) \stackrel{\text { def }}{=} \mathbf{E}^{i} f\left[\Phi_{\zeta}(t, 0) x\right]
$$

where $\mathbf{E}^{i}$ denotes expectation condition on the initial mode being the $i$-th. This defines the expectation semigroup for the $P M^{3} D$ case.

Setting up the conditional Kolmogorov backward equation (conditioned on the initial mode) proceeds as in the previous section. There is however a potential problem with the averaging over the initial state, stemming from the different dimensions in various modes. What is needed is a compatibility restriction for the class of functions $f(\zeta, \mathbf{x})$ and initial states $\mathbf{x}$. We impose

$$
f\left(i, x_{i}\right)=f\left(j, x_{j}\right)
$$

implying that the observable $f$ yields the same value, regardless the mode. This implies at once that the mode itself is not detectable from one simple observation. Another added difficulty is the incorporation of the pseudo continuity in the Kolmogorov equation.

4.1. Alternating $P M^{3} D$ Systems. For the two mode system we use the binary $(0,1)$ notation instead of $(1,2)$. Following the ideas in the previous section, taking the 
quasi-stationarity and the compatibility into account, one obtains the coupled PDE's

$$
\begin{aligned}
& \frac{\partial F^{0}}{\partial t}=\frac{\partial F^{0}}{\partial x_{0}}\left(A_{0} x_{0}+b_{0} u\right)+\pi_{0}^{1}\left[F^{1}\left(S_{10} x_{0}, t\right)-F^{0}\left(x_{0}, t\right)\right] \\
& \frac{\partial F^{1}}{\partial t}=\frac{\partial F^{1}}{\partial x_{1}}\left(A_{0} x_{1}+b_{1} u\right)+\pi_{1}^{0}\left[F^{0}\left(S_{01} x_{1}, t\right)-F^{1}\left(x_{1}, t\right)\right]
\end{aligned}
$$

4.2. An Example: A Pebble Elevator Belt. Consider a pebble on a conveyor belt, moving with velocity $v$. For some time, the pebble may stick to the band and move with it. At other times the pebble may be airborne, and initiate a free fall, rolling back on the transport band until it sticks again (Figure 5).

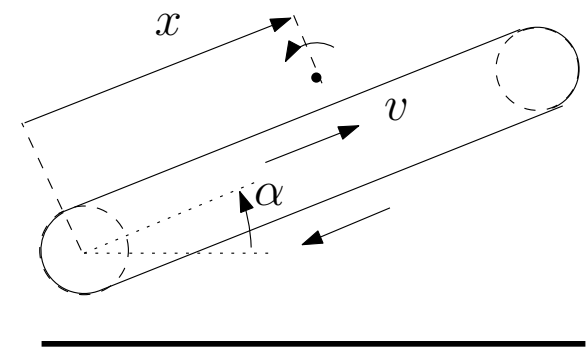

FIG. 5. The conveyor belt

Letting $x$ be the distance from the bottom of the band, the first mode (sticking) obeys

$$
\dot{x}=v
$$

In the rolling mode, the dynamics is modeled by

$$
\begin{aligned}
& \dot{x}=y \\
& \dot{y}=-g \sin \alpha
\end{aligned}
$$

Let $g \sin \alpha=1$ to minimize notation. We neglect impulsive behavior at the transitions, so that the switching rules are

$$
\begin{gathered}
\text { stick } \rightarrow \text { slip }: x \rightarrow[x, v]^{T} . \\
\text { slip } \rightarrow \text { stick }:[x, y] \rightarrow[x]^{T} .
\end{gathered}
$$

Note that the stick to slip transition is actually not quasi-continuous due to inertia (conserved velocity), but the problem is easily circumvented. Consider the class of functions having only position $(x)$ as their argument. This is a compatible class since $f(\zeta, \mathbf{x})=f\left(0, x_{0}\right)=f\left(1, x_{1}\right)=f(x)$, and at $t=0, x_{0}=x_{1}=x$. The resulting Kolmogorov equations are (suppressing the time argument)

$$
\begin{aligned}
\frac{\partial F^{0}\left(x_{0}\right)}{\partial t} & =v \frac{\partial F^{0}\left(x_{0}\right)}{\partial x_{0}}+\lambda\left[F^{1}\left(x_{0}, v\right)-F^{0}\left(x_{0}\right)\right] \\
\frac{\partial F^{1}\left(x_{1}, y_{1}\right)}{\partial t} & =y \frac{\partial F^{1}\left(x_{1}, y_{1}\right)}{\partial x_{1}}-\frac{\partial F^{1}\left(x_{1}, y_{1}\right)}{\partial y_{1}}+\lambda\left[F^{0}\left(x_{1}\right)-F^{1}\left(x_{1}, y_{1}\right)\right] .
\end{aligned}
$$


How does one make sense of $F^{1}(x, y, t)$ for an initial condition $F^{1}(x, y, 0)=f(x, y)$ involving $y$ ? The interpretation $F^{1}(x, y, t)=\mathbf{E} f(x(t), y(t))$ runs into trouble since $y$ is not a state variable in the stick mode. However, it is possible to define here the $y$ as an output variable (with the trivial $y=v$ in this mode.) Equivalently, one may assume that upon measurement (expressing $y(t)$ ), the system switches to the the slip mode if it wasn't in there. This idea looks somewhat analogous to the collapse of the wave function in quantum mechanics.

In order to get an idea of the behavior of the solution to the above system, we first set $\lambda=0$. In this case, the general solution is for arbitrary functions $g^{0}(\cdot)$ and $g^{1}(\cdot, \cdot)$

$$
\begin{aligned}
F^{0}(x, t) & =g^{0}(x+v t) \\
F^{1}(x, y, t) & =g^{1}\left(2 x+y^{2}, t-y\right) .
\end{aligned}
$$

If we let $F^{0}(x, 0)=F^{1}(x, y, 0)=f(x)$ then $F^{0}(x, t)=f(x+v t)$ and $F^{1}(x, y, t)=$ $f\left(x+y t-\frac{1}{2} t^{2}\right)$. Consider next $\lambda \neq 0$. Because of switchings, we now expect the behavior, for the same initial condition, to be asymptotically given by

$$
\begin{aligned}
F^{0}(x, t) & \rightarrow f(x+k t) \\
F^{1}(x, y, t) & \rightarrow f(x+k t-\epsilon(y)),
\end{aligned}
$$

for some $k<v$. This motivates us to start from the asymptotic (for sufficiently large $t$ ) assumption $F^{1}(x, y, t) \sim F^{0}\left(x+\frac{y}{\lambda}-\frac{1}{\lambda^{2}}, t-\frac{1}{\lambda}\right)$. In fact, this can be derived by taking the expectation with respect to first switching time from the slip mode (1) to the stick mode $(0)$.

Substituting this into the PDE for $F^{0}$ gives, upon approximating $F^{1}(x, v)-F^{0}(x)$ by $\frac{\partial F^{0}}{\partial x}\left(\frac{y}{\lambda}-\frac{1}{\lambda^{2}}\right)-\frac{\partial F^{0}}{\partial t}\left(\frac{1}{\lambda}\right)$, the simple transport equation

$$
\frac{\partial F^{0}(x)}{\partial t}=\left(v-\frac{1}{2 \lambda}\right) \frac{\partial F^{0}(x)}{\partial x}
$$

Compute its solution for initial condition $F^{0}(x, 0)=f(x)$. Denote this (approximate) solution by $F_{0}^{0}(x, t)$. Next substitute this solution into the PDE for $F^{1}(x, y, t)$, which can now easily be solved, to yield $F_{1}^{1}(x, y, t)$. In turn use this in the first PDE, which is now solved exactly to yield $F_{1}^{0}(x, t)$. Iterating, one obtains

$$
\cdots \rightarrow F_{k}^{0}(x, t) \rightarrow F_{k+1}^{1}(x, y, t) \rightarrow F_{k+1}^{0}(x, t) \rightarrow \cdots
$$

We compute this for the initial conditions $f(x)=x$ and $f(x)=x^{2}$. If the process converges, its limit is respectively $\mathbf{E}(x(t) \mid x(0)=0)$ and $\mathbf{E}\left(x^{2}(t) \mid x(0)=0\right)$ conditioned on the initial mode, from which thus the variance may be obtained. In figure 6 , the mean and variance are shown for the process, with $\lambda=v=1$ after one iteration (i.e., 
respectively $F_{1}^{0}$ and $F_{1}^{1}$.) The three solid curves give the expected position of the pebble, enveloped in its 1- $\sigma$ RMS bounds as function of $t$ for a pebble starting in the stick mode at $x=0$. The dashed curves show this for the slip mode.

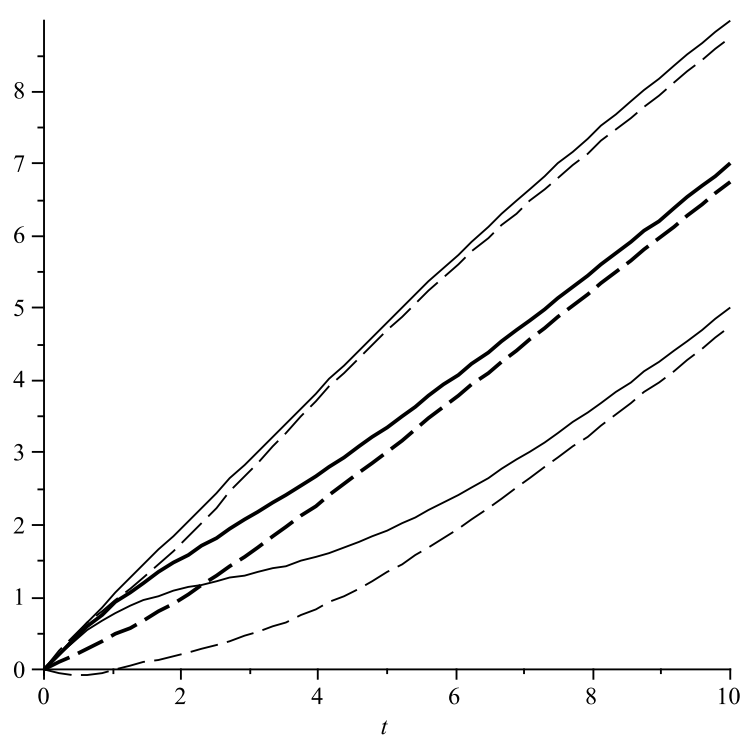

FIG. 6. Expected value of $x(t)$ and its $R M S$ bounds.

We should add that the model of purely random transitions between the stick and the slip phase is somewhat unrealistic. The probability of a change from stick to slip (rolling), is probably higher than for slip to stick. It would not complicate the model much more to add this realism.

5. Conclusions. We introduced a new class of hybrid systems, having modes with internal description of varying dimension. The notion of pseudo-continuity was introduced for the transitions at switching events. We showed several examples and motivated such systems, including approximations to nonlinear systems, either from the point of view local balancing, or as a piecewise linearized system and in remote control with one-way communication.

In the second part of the paper, we discussed a version of the problem where the switches occur at random times, derived the backward Kolmogorov equation and discussed in particular the fast switching diffusion approximation. For linear modes, the Lie-algebraic structure can be exploited to derive vibrational stabilization criteria. Finally, the last part on $P M^{3} D$ systems, showed how the added difficulty of solving coupled PDE's in different numbers of variables can be dealth with. We illustrated this with a simplified model of a conveyor belt carrying pebbles. The exposed model and methodology may be relevant in the study of nanoscale motions (Brownian motor). An approach towards extending these results for randomly switched systems with 
delays is suggested in [23].

\section{REFERENCES}

[1] M.S. Branicky, V.S. Borkar, and S. Mitter, A Unified Framework for Hybrid Control Theory: Model and Optimal Control Theory. IEEE Trans. Auto. Control, 43:1(1998), pp. 31-45.

[2] R.W. Brockett, Hybrid Models for Motion Description Control Systems. In: Essays on Control: Perspectives in the Theory and its Applications, H.L. Trentelman and J.C. Willems (Eds.), Birkhäuser, 1993.

[3] D. Liberzon and R.W. Brockett, Nonlinear Feedback Systems Perturbed by Noise: SteadyState Probability Distributions and Optimal Control. IEEE Trans. on Auto. Control, 45:6(2000).

[4] A. Buonocore, L. Caputo, E. Pirozzi, And L.M. Ricciardi, On a pulsating Brownian motor and its characterization. Mathematical Biosciences, 207(2007), pp. 387-401.

[5] A. Tözeren and S.W. Byers, New Biology for Engineers and Computer Scientists, Pearson Prentice-Hall, 2004.

[6] D. Yeung and E. I. Verriest, A Stochastic Approach to Optimal Switching between Control and Observation, Proceedings of the 45-th IEEE Conference on Decision and Control, San Diego, CA, 2006, pp. 2655-2660.

[7] L. Arnold, Stochastic Differential Equations: Theory and Applications, Wiley,1974.

[8] L. Arnold, H. Crauel, And V. Winstutz, Stabilization of Linear Systems by Noise, SIAM J. Control and Optimization, 21:3(1983), pp. 451-461.

[9] M.A. Berger and A.D. Slonn, A Method of Generalized Characteristics, Memoirs of the AMS, 38:266(1982).

[10] Ph. Blanchard, Ph. Combe, M. Sirugue, and M. Sirugue-Collin, Jump Processes related to the Two Dimensional Dirac Equation, in: Stochastic Processes, Mathematics and Physics II, Albeverio, Blanchard and Streit (eds.), Lecture Notes in Mathematics, No. 1250, Springer-Verlag, 1985.

[11] B. Gaveau, T. Jacobson, M. Kac, and L.S. Schulman, Relativistic Extension of the Analogy between Quantum Mechanics and Brownian Motion, Physical Review Letters, 53:5(1984), pp. 419-422.

[12] P.G. Hoel, S.C. Port And C.J. Stone, Inroduction to Stocahstic Processes, Houghton Mifflin, Boston, 1972.

[13] T. Jacobson, And L.S. Schulman, Quantum Stochastics: the Passage from a Relativistic to a Nonrelativistic Path Integral, J. Phys. A: Math. Gen., 17(1984), pp. 375-383.

[14] M. KAC, A Stochastic Model related to the Telegrapher's Equation, Rocky Mountain Journal of Mathematics, 4:3(1974), pp. 497-509.

[15] R.Z. Khasminskit, Stochastic Stability of Differential Equations, Sijthof \& Noordhoff, Alphen aan den Rijn, 1985.

[16] S.M. Meerkov, Principle of Vibrational Control: Theory and Applications, IEEE Transactions on Automatic Control, 25(1980), pp. 755-762.

[17] M.A. Pinsky, Lectures on Random Evolutions, World Scientific, 1991.

[18] E.I. Verriest, On a Hyperbolic PDE Describing the Forward Evolution of a Class of Randomly Switched Systems and Connections with the Kolmogorov Equation and QED, Proceedings of the 1990 Conference on Information Systems and Sciences, Princeton University, Princeton, NJ, 1990.

[19] E.I. Verriest, On a Hyperbolic PDE Describing the Forward Evolution of a Class of Randomly Alternating Systems, Proceedings of the 29th IEEE Conference on Decision and Control, 
Honolulu, HI, pp. 2147-2148, 1990.

[20] J.L. Willems, Stability Criteria for Stochastic Systems with Colored Multiplicative Noise. Acta Mechanica, 23(1975), pp. 171-178.

[21] J.L. Willems, Stability of Higher Order Moments for Linear Stochastic Systems, IngenieurArchiv, 44(1975), pp. 123-129.

[22] H. Sussmann, (1999). A Maximum Principle for Hybrid Optimal Control Problems. Proc. 38th Conference on Decision and Control, Phoenix, AZ, 425-430.

[23] E.I. Verriest, A Hyperbolic PDE describing the Evolution of a Class of Randomly Switched Delay Systems and Stochastic Stabilization. Proceedings of the 6-th IFAC Workshop on Time-Delay Systems, L'Aquila, Italy, 2006.

[24] E.I. Verriest, Multi-mode Multi-dimensional Systems, Proceedings of the 17th International Symposium on Mathematical Theory of Networks and Systems, Kyoto, Japan, pp. 12681274, 2006.

[25] E.I. Verriest And W.S. Gray, Geometry and Topology of the State Space via Balancing, Proceedings of the 17th International Symposium on Mathematical Theory of Networks and Systems, Kyoto, Japan, pp. 840-848, 2006. 